\title{
Operative laparoscopy; is it a safe option in pregnancy?
}

\author{
A. Rauf $\cdot$ P. Suraweera $\cdot$ S. De Silva
}

Received: 16 March 2008 / Accepted: 25 June 2008 /Published online: 24 July 2008

(C) Springer-Verlag 2008

\begin{abstract}
We report a case of an ovarian cyst in pregnancy complicated by torsion of the cyst requiring urgent surgical intervention at 13 weeks of gestation. The case was successfully managed by laparoscopic ovarian cystectomy without any maternal or fetal complications. Thus proving that operative laparoscopy in pregnancy is a safe and feasible option. Approximately $0.2 \%$ of pregnant women require intra-abdominal general surgery during pregnancy. Benefits of laparoscopic surgery compared to the traditional open surgery are well recognised; notably, a shorter hospital stay and a reduced rate of post-operative complications. Pregnancy poses unique surgical and anaesthetic problems due to altered physiology and anatomy in pregnancy. The effect of the pneumoperitoneum on the maternal haemodynamic status, risk of uterine/fetal damage by trocar entry, risk of miscarriage and preterm labour are some serious concerns that may limit the feasibility and safety of operative laparoscopy in pregnancy. In the past few decades, there has been increasing evidence emerging in the medical literature supporting the safety of operative
\end{abstract}

\section{A. Rauf}

Obstetrics \& Gynaecology,

Royal Wolverhampton Hospitals NHS Trust,

New Cross Hospital Wednesfield Road,

Wolverhampton WV10 0QP, UK

P. Suraweera $(\square)$

Obstetrics \& Gynaecology, Mid-Staffordshire General Hospital,

Weston Rd,

Stafford ST16 3SA, UK

e-mail: prabathbagi@hotmail.com

S. De Silva

Russells Hall Hospital,

Dudley DY1 2HQ, UK laparoscopy in pregnancy. The second trimester is considered to be the safest period in terms of least risk to the fetus and also seems more favourable for the technical aspect of the laparoscopic procedures. Hasson's method of laparoscopic entry technique is prudent to avoid possible uterine injury. Although absolute safety of laparoscopic surgery during pregnancy in humans is yet to be established, presently, operative laparoscopy by an experienced laparoscopist is considered a safe and effective alternative to traditional laparotomy. Prospective randomised controlled studies in this clinical territory are lacking.

Keywords Laparoscopy · Pregnancy · Adnexal mass · Pregnancy complications

\section{Case report}

A 22-year-old woman in her first pregnancy attended her dating scan at 11 weeks and incidentally revealed a multiloculated cystic mass in the left ovary measuring $87 \times 74 \times 41 \mathrm{~mm}$. She gave a background history of gradually worsening right sided lower abdominal pain for about 6 months.

Ovarian tumour markers were not done as they have a limited value in evaluating ovarian cysts in pregnancy; because, during pregnancy, tumour markers such CA 125, beta-hCG, alpha-fetoprotein and inhibin levels are raised due to desidual and placental synthesis.

Conservative management with serial follow up scans was agreed upon with provision for surgery in the case of cyst complication or suspicion of malignancy. Should elective surgery be necessary, 14 weeks of gestation and beyond in the second trimester is considered to be the optimal time period in a bid to mitigate the risk of fetal loss. 
She was admitted as an emergency at 13 weeks of gestation with a history of acute severe colicky right side lower abdominal pain and vomiting. On examination, she was in significant distress and tachycardic but was normotensive and apyrexial. Marked tenderness on a diffuse area of the lower abdomen particularly on the right side with guarding rigidity and rebound tenderness were noted. Pain was little amenable to parenteral morphine administration.

Inflammatory markers including white cell count and C-reactive protein were both within normal reference range. The diagnosis of acute appendicitis was considered unlikely after assessment by the surgical discipline but could not be completely ruled out. Pelvic ultrasound scan during acute admission confirmed the presence of the previously seen ovarian cyst with some free fluid in the Pouch of Douglas.

With a background history of a large ovarian cyst, a corroborative history and examination findings made ovarian cyst complication (torsion/haemorrhage/rupture) the most likely diagnosis. Hence, urgent laparoscopy was performed after informed consent and adequate counselling about possible fetal complications.

Closed trocar entry technique was employed with carbon dioxide pneumoperitoneum achieving a pressure of $15 \mathrm{mmHg}$. A twisted left ovarian cyst was noted. Laparoscopic cystectomy was performed successfully after untwisting the cyst. The time taken for the procedure was $45 \mathrm{~min}$.

Fetal viability was confirmed post-operatively. She had an uneventful post-operative recovery and the total length of hospital stay was $26 \mathrm{~h}$. Histological assessment confirmed a serous cyst-adenoma.

The subsequent antenatal course was unremarkable. A live healthy female baby weighing $3.2 \mathrm{~kg}$ was delivered vaginally at 39 weeks of gestation.

\section{Discussion}

Introduction

Approximately $0.2 \%$ of pregnant women require intraabdominal general surgery during their pregnancies.

Although the concepts of laparoscopic surgery in pregnancy were put forth over a century ago, its acceptance as a useful procedure has evolved only recently within the last 15 years or so. In 1901, von Ott from St. Petersburg described examination of the abdominal cavity of a pregnant woman through a culdoscopic incision using a head mirror to reflect light. Since the 1940's, laparoscopy has been used for tubal sterilization and the surgical management of ectopic pregnancies [11, 21].

Until recently, pregnancy was considered an absolute contraindication for laparoscopic surgery. As a result of a growing number of reports in the literature suggesting its safe use in pregnancy, operative laparoscopy in pregnancy has been performed at increasing frequency in many centres across the world in the last couple of decades. The challenging nature of laparoscopic surgery during pregnancy accounts for a proportion of the reluctance among surgeons to take this route.

\section{Benefits of laparoscopic surgery}

The major advantages of laparoscopic surgery during pregnancy, over traditional laparotomy, are more or less the same as in a non-pregnant woman. These include smaller abdominal incisions resulting in less post-operative pain, less infection and a reduced incidence of incisional hernias.

Further benefits of minimally invasive laparoscopic surgery are early return of gastrointestinal activity (due to less manipulation of the bowel during surgery), minimal post-operative adhesions and fewer incidences of intestinal obstruction. Laparoscopy is also associated with shorter hospitalization time and prompt return to regular life.

Additional benefits of laparoscopic surgery specific to pregnancy are the reduced risk of fetal depression by narcotic use [7] due to fewer requirements for strong analgesics, and more rapid post-operative recovery with resultant earlier mobilization, which helps minimize the increased risk of thromboembolism with pregnancy and surgery [17].

Constraints and risks of laparoscopic surgery in pregnancy

Concerns regarding the deleterious effects of laparoscopic surgery on the mother and fetus include possible maternal haemodynamic changes and instability secondary to decreased venous return and cardiac output. Also, an increase in intra-abdominal pressure may lead to decreased uterine blood flow and increased intrauterine pressure. All of these changes could result in fetal hypoxia [10, 14, 15]. The acidosis experienced in patients undergoing a carbon dioxide pneumoperitoneum could be potentiated in the fetus, leading to respiratory acidosis in the mother of the

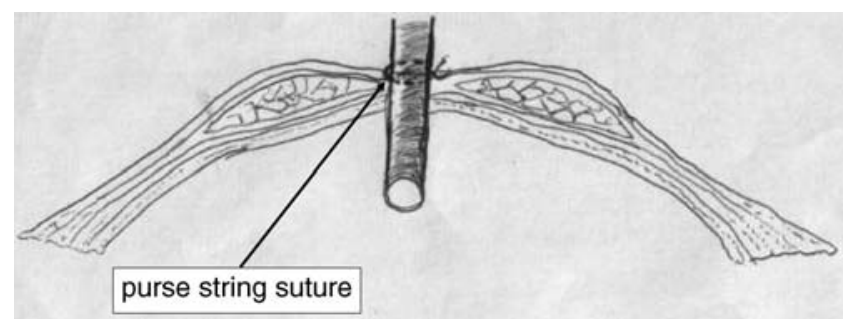

Fig. 1 Hasson technique through intraumbilical incision 
fetus [15, 17-19]. Inadvertent uterine and fetal injury by Veress needle and primary trocar entry is another area of concern which renders the procedure risky, particularly in the third trimester.

The fetal and maternal risk factors are governed by the stage of the pregnancy. During the first trimester of pregnancy, the major risks are increased risk of miscarriage $(12 \%)$ and possible teratogenesis [23]. Risk of miscarriage is possibly due to disturbance to the corpusluteum as the developing pregnancy is hormonally dependent on it in the first trimester. Laparoscopic surgery in the third trimester carries increase risk of preterm labour and poses unique technical difficulties and maternal risks due to the extent of the space occupied by the gravid uterus. Detail discussion of safety and technical aspects of operative laparoscopy in the third trimester is, however, beyond the scope of this article.

Conversely, in the second trimester, the risk of miscarriage, teratogenesis and preterm labour are minimal and the gravid uterus does not create an obstruction [1, 6, 8]. For these reasons, the second trimester is considered the safest and most pragmatic time period for surgery in pregnant patients $[5,13,16,22]$.

However, the absolute safety of laparoscopic surgery during pregnancy in humans is yet to be established as no controlled studies have been reported yet. In a retrospective case control study, Curet et al. [4] compared 16 patients who underwent laparoscopic surgery with 18 control patients undergoing open laparotomy during their first or second trimester. There was no difference between laparotomy and laparoscopy outcomes. Gouldman et al. [12] reported no post-operative complications to mother or fetus in their series of laparoscopic cholecystectomies. Reedy et al. [20] analyzed 2,181 laparoscopies and 1,522 laparotomies performed in singleton pregnancies, between the fourth and twentieth weeks of gestation. There was no difference in laparoscopy and laparotomy in the rate of malformation and cumulative infant survival rate. Mazze and Kallen [17] published the largest study involving 5,405 pregnant patients and found no increased incidence of still births or congenital anomalies in each trimester.

\section{Precautions}

Some suggested precautions to be taken during performing laparoscopy in pregnancy include close intra-operative fetal monitoring by transvaginal ultrasound, positioning the patient in the left lateral decubitus position to prevent aorto-caval compression and meticulous attention on air way management to circumvent the increased risk of aspiration due to raised intra-abdominal pressure.

A Hasson open laparoscopic technique seems prudent in order to prevent inadvertent puncture of the uterus especially in advanced gestations. The technique involves the introduction of primary trocar through a $2-3-\mathrm{cm}$ long superficial incision after dissection of fascia and incision of the peritoneum and finally the pneumoperitoneum is secured with a purse string suture. However, intraumbilical Veress technique to create pneumoperitoneum is feasible with sufficient care and the expertise as in our case (Fig. 1).

It may be prudent to avoid intra-abdominal pressure rising beyond $15 \mathrm{mmHg}$ which would pose technical difficulty in ventilating.

Maternal end tidal volume $\mathrm{CO}_{2}$ should be monitored and kept within the normal range. Tocolytic agents need not be used prophylactically, but can be administered if the uterus starts contracting. Should elective surgery be embarked upon, the second trimester seems the ideal time for the successful completion of laparoscopic surgery with the least risk of maternal and fetal adverse effects. Moreover, an experienced theatre team and appropriate equipment are a prerequisite to the safety and success of operative laparoscopy in pregnancy $[1,2,3,7-9,13,22]$.

\section{Conclusion}

The second trimester is traditionally considered to be the optimal time for laparoscopic procedures. The success and risk profile of laparoscopic surgery in pregnancy are skilldependent. Proper precautions should be embarked on to obviate the maternal and fetal risks arising from the anaesthesia and surgical procedure. The evidence so far available, based on retrospective cohort studies and case reports, suggest that it is safe and beneficial for the mother and the fetus. Nonetheless, absolute safety of laparoscopic surgery during pregnancy and the long-term effects have yet to be fully ascertained. Although randomised controlled studies are imperative to yield the answer to many of these clinical uncertainties, the feasibility is critically limited due to ethical reasons.

\section{References}

1. Arvidsson D, Gerdin E (1991) Laparoscopic cholecystectomy during pregnancy. Surg Lapafosc Endosc 1:193

2. Barnard JM, Chaffin D, Droste S et al (1995) Fetal response to carbon dioxide pneumoperitoneum in the pregnant ewe. Obstet Gynecol 85:669

3. Comaitalo JB, Lynch D (1994) Laparoscopic cholecystectomy in the pregnant patient. Surg Laparosc Endosc 4:268

4. Curet MJ, Allen D, Josloff RK et al (1996) Laparoscopy during pregnancy. Arch Surg 31:546-551

5. Curet MJ, Vogt DM, Schob O et al (1996) Effects of $\mathrm{CO}_{2}$, pneumoperitoneum in pregnant ewes. J Surg Res 63:339

6. Duncan PG, Pope WDB, Cohen MM et al (1986) Fetal risk of anaesthesia and surgery during pregnancy. Anaesthesiology 64:790-794 
7. Duncan PG, Pope WDB, Cohen MM et al (1986) The safety of anaesthesia and surgery during pregnancy. Anesthesiology 64:790-794

8. El-Minawi MF, Wahbi O, El-Bagouri ES et al (1992) Physiologic changed during $\mathrm{CO}_{2}$ and $\mathrm{N}_{2} \mathrm{O}$ pneumoperitoneum. Am J Surg $163: 186$

9. Ghumann E, Barry M, Grace PA (1997) Management of gallstones in pregnancy. Br J Surg 84:1646

10. Glasgow RE, Visser BC, Harris HW et al (1998) Changing management of gallstone disease during pregnancy. Surg Endosc $12: 241$

11. Gordon AG, Magos AL (1989) The development of laparoscopic surgery. Baillere's Clin Obstet Gynecol 3:429-448

12. Gouldmann JW, Sticca RP, Rippon MB et al (1998) Laparoscopic cholecystectomy in pregnancy. Am Surgeon 64:93-97

13. Halpern NB (1998) Laparoscopic cholecystectomy in pregnancy: a review of published experiences and clinical considerations. Sermin Laparosc Surg 5:129

14. Kammerer WS (1979) Nonobstetric surgery during pregnancy. Med Clin North Am 63:1157

15. Lanzafame R (1995) Laparoscopic cholecystectomy during pregnancy. Surgery 118:627
16. Levy T, Dicker D, Shalev J et al (1995) Laparoscopic unwinding of hyperstimulated ischemic ovaries during the second trimester of pregnancy. Hum Reprod 10:1478-1480

17. Mazze RI, Kallen B (1989) Reproductive outcome after anesthesia and operation during pregnancy: a registry study of 5,405 cases. Am J Obstet Gynecol 161:1178-1185

18. McKellar DP, Anderson CT, Boynton CJ et al (1992) Cholecystectomy during pregnancy without fetal loss. Surg Gynecol Obstet $174: 465$

19. Posta CG (1995) Laparoscopic surgery in pregnancy: report on two cases. J Laparoendosc Surg 6:203

20. Reedy MB, Kallen B, Keuhl TJ (1997) Laparoscopy during pregnancy: a study of five fetal outcome parameters with use of the Swedish Health Registry. Am J Obstet Gynecol 177:673-679

21. Rock JA, Warshaw JR (1994) The history and future of operative laparoscopy. Am J Obstet Gynecol. 170:7-11

22. Soper NJ, Hunter JG, Petri RH (1992) Laparoscopic cholecystectomy during pregnancy. Surg Endosc 6:115

23. Soriano D, Yefet Y, Seidman DS, Goldenberg M, Mashiach S, Oelsner G (1991) Laparoscopy versus laparotomy in the management of adnexal masses during pregnancy. Fertil Steril 71:955960 